\title{
Evaluation of the Microstructure, Micro Hardness, Electrical Resistivity and Ultrasonic Properties of 6201Alloy Containing Lanthanum
}

\author{
*Amira Nassar ${ }^{1}$ and L. Abd El-Latif ${ }^{2}$ \\ ${ }^{1}$ Solid State physics Department, National research Centre, 33 El Bohouth \\ St.12622 Dokki, Giza, Egypt. \\ ${ }^{2}$ Ultrasonic Laboratory, National Institute for Standards, Tersa Street, \\ P.O. Box 136, El-Haram, El-Giza 12211, Egypt. \\ *E-mailfatmohmar@yahoo.com
}

This research is to investigate the influence of adding different concentrations of $(0.0109,0.0185$ and $0.037 w t \%)$ La on the microstructure, electrical resistivity, Vickers micro hardness (Hv) and ultrasonic properties on 6201 aluminum alloy comparing to (6201alloy +0.021wt\%TiB) used in an electrical industry. The results showed that the (6201alloy+0.037wt\% La) was suitable materials for using electrical conductor than alloys added with0.0185wt\%La and 0.0109wt\% La. It was also found that Vickers micro hardness (Hv) measured mechanically and electrical resistivity for the alloys contaning $\mathrm{La}$ has the same trend to those determined from ultrasonic calculations. It was also found that the structural changes inside the matrix of the alloys play an important role in the ultrasonic wave propagation and also in controlling the electrical resistivity and the alloys strength.

\section{Introduction}

In electrical applications, the commercially pure aluminum possesses the highest electrical conductivity among aluminum conductors. Its application is only restricted due to its low strength [1]. Series of aluminum alloys containing Mg and $\mathrm{Si}$ as major addition elements show a high strength-to- density ratio, a good electrical conductivity ability and a superior corrosion resistance. In recent years, the aluminum alloy wires made from AA6201 with high tensile strength and medium electrical conductivity are widely used in electrical engineering field. The common disadvantage of the aluminum alloy wires made from AA6201 is "the higher tensile strength, the lower electrical conductivity” [2, 3]. In these alloys, $\mathrm{Mg}$ and $\mathrm{Si}$ combine to form the chemical compound $\mathrm{Mg}_{2} \mathrm{Si}$ (magnesium-silicide), which is considered as the primary hardening phase $[4,5]$.

Grain refining is one of the predominant techniques in controlling the strength and toughness and also for subsequent secondary processing of castings. Grain refinement by addition of grain-refiners like titanium boride referred to as inoculation [6] is the most popular due to its simplicity. Sometimes a master alloy of $\mathrm{Al}-\mathrm{TiB}$ is made and used subsequently to grain refining. 
In recent studies, the rare earth $\mathrm{La}$ was added into the $\mathrm{Al}$-alloy to investigate its effect on the Al. However the additional La can improve the welding properties of aluminum alloy (Al-Mg-Si) by promoting the diffusion of $\mathrm{Mg}$, $\mathrm{Si}$ in base metal and decreasing conglomeration of impurities in unit area of grain boundary. Apart from these, La is an effective grain refinement additive during the casting process. A proper amount of La could modify the microstructure of the $\mathrm{Al}$ alloys and improve its mechanical property, electrical conductivity and thermal-resistance [7, 8].

In a wide range of different ultrasonic nondestructive methods, the evaluation of material properties by ultrasonic measurements is introduced as a quality control method, by which the need for conventional destructive tests would be minimized. It has been proven that indirect assessment of the microstructure by ultrasonic velocity or attenuation measurements is possible. However, ultrasonic is based on a simple principle of physics in which any wave will affect by the medium through which it travels. Thus, changes in the micro structural features which control various mechanical properties can be sensed by ultrasonic measurements. $[9,10]$. Therefore, establishing a relationship between the micro structural parameters and ultrasonic evaluation results may be useful for fast and nondestructive quality control of the materials.

The main object of this work is to compare the microstructure, electrical resistivity, ultrasonic properties and Vickers micro hardness behavior of 6201alloy in the presence of different concentrations of $\mathrm{La}$ and in the presence of TiB which used in an electrical industry.

\section{Experimental}

The base metal 99.6\% aluminum was melted in a graphite crucible using heating furnace. The required amounts of (0.6-0.9wt \%) Mg and (0.5-0.9wt \%) Si was added into the molten to produce 6201 alloy used in electrical industry. Each of the master alloy of Al-TiB and Al-La with different percentages were added to the above melt and stirred with a graphite rod to produce a series of modifying alloys namely $(6201+0.021 \mathrm{wt} \% \mathrm{TiB}, 6201+0.0185 \mathrm{wt} \% \mathrm{La}, 6201+0.037 \mathrm{wt} \% \mathrm{La}$ and $6201+0.0109 \mathrm{wt} \% \mathrm{La})$. The melts were poured into iron molds to get casting alloys in the form of cylinder (200 $\mathrm{mm}$ in length and $20 \mathrm{~mm}$ in diameter).Specimens were cut from the as-cast rod for different measurements.

All the specimens for microstructure characterization were cut from the cast cylinder then polishing and etching by hydrofluoric acid. The micro structural analysis of the samples was carried out by using Environmental Scanning Electron Mcroscope (FEI-S). The phases of the samples were analyzed by the EDS system of SEM and X-Ray Diffractometer (XRD) system EMPYREAN by Cuk ${ }_{\alpha}$ radiation with a wavelength, $\lambda$, of $1.54060 \mathrm{~A}^{\circ}$.

Micro hardness (Hv) measurements are evaluated by using a Vickers micro hardness tester with a load of $1.96 \mathrm{~N}$ for $5 \mathrm{~s}$. 
For the measurement of the electrical resistivity $(\rho)$, the samples were prepared in form of discs $2 \mathrm{~cm}$ diameter and $0.2 \mathrm{~cm}$ thickness. The surfaces of the samples were polished and having two parallel faces. The direction of measurement was always at contact surface area of the sample. Resistance (R) measurement was carried out using Hioki 3522-50LCR Hitester (Bridge) at room temperature, then the electrical resistivity $(\rho)$ of the samples was calculated from the following eq.(1)

$$
\rho=R A / L(1)
$$

where $\mathrm{L}$ is the thickness of the sample in $\mathrm{cm}$, and A is the surface area of the sample in $\mathrm{cm}^{2}$.

For ultrasonic measurement, discs with dimensions of $10 \mathrm{~mm} \times 15 \mathrm{~mm} \times$ $15 \mathrm{~mm}$ were polished to obtain smooth and uniform surface.

The density (D) of the samples was measured by applying Archimedes principle at room temperature using toluene as an immersion liquid according to the formula:

$$
\mathrm{D}=\mathrm{D}_{\ell}\left[W_{\mathrm{d}}\left(W_{\mathrm{a}}+W_{\ell}\right)\right]
$$

Where $\mathrm{D}_{\ell}$ is the density of toluene, $W_{\mathrm{a}}$ and $W_{\ell}$ are the sample weights in air and liquid, respectively.

The ultrasonic wave velocity measurements were carried out by applying the pulse-echo technique. The elapsed time between the initiation and the receipt of the pulse appearing on the screen of a flaw detector (GE model: USN60) was determined. The ultrasonic wave velocity was therefore, calculated by dividing the round trip distance (twice the thickness of the sample) by the elapsed time according to equation (3);

$$
\mathrm{V}=2 \mathrm{X} / \Delta \mathrm{t}
$$

where $\mathrm{X}$ is the sample thickness and $\Delta \mathrm{t}$ is the time interval.

All ultrasonic wave velocity measurements were carried out at room temperature using Karl Deutsch transducer S24 HB4 with fundamental frequency of $4 \mathrm{MHz}$ to measure longitudinal ultrasonic wave velocity, and Krautkramer transducer K2NY with fundamental frequency of $2 \mathrm{MHz}$ for measuring shear ultrasonic wave velocity. The estimated uncertainty (Type A) in ultrasonic wave velocity measurements was $\pm 40 \mathrm{~m} / \mathrm{s}$ for longitudinal ultrasonic wave velocity $\left(\mathrm{V}_{\mathrm{L}}\right)$ and $\pm 15 \mathrm{~m} / \mathrm{s}$ for shear ultrasonic wave velocity (Vs).

Young's modulus (E), shear modulus (G), longitudinal modulus (L), bulk modulus $(\mathrm{K})$, microhardness $(\mathrm{H})$ and Poisson's ratio $(\sigma)$ of the investigated samples have been determined from the measured ultrasonic wave velocities and density measurement as equations: $[11,12]$. 


$$
\begin{gathered}
\mathrm{L}=\rho V^{2} \mathrm{~L} \\
\mathrm{G}=\rho V_{\mathbf{S}}^{\text {घ. }} \\
\mathrm{K}=\mathrm{L}-4 / 3 \mathrm{G} \\
\mathrm{E}=2 \mathrm{G}(1+\sigma) \\
\sigma=\mathrm{L}-2 \mathrm{G} / 2(\mathrm{~L}-\mathrm{G}) \\
\mathrm{H}=\mathrm{E}(1-2 \sigma) / 6(1+\sigma)
\end{gathered}
$$

\section{Results and Discussion}

\section{1. Microstructure characteristic}

The microstructures of 6201 alloy with $\mathrm{TiB}$ and $\mathrm{La}$ addition in various proportions (i.e, $0.021 \mathrm{wt} \% \mathrm{TiB}, 0.0185 \mathrm{wt} \% \mathrm{La}, 0.037 \mathrm{wt} \% \mathrm{La}$ and $0.0109 \mathrm{wt} \%$ La) were characterized by SEM as shown in Figs.(1 a, b, c and d), respectively. It is evident from these figures that grains are fine, spherical and rod-like shaped within the grain body and along grain boundaries.
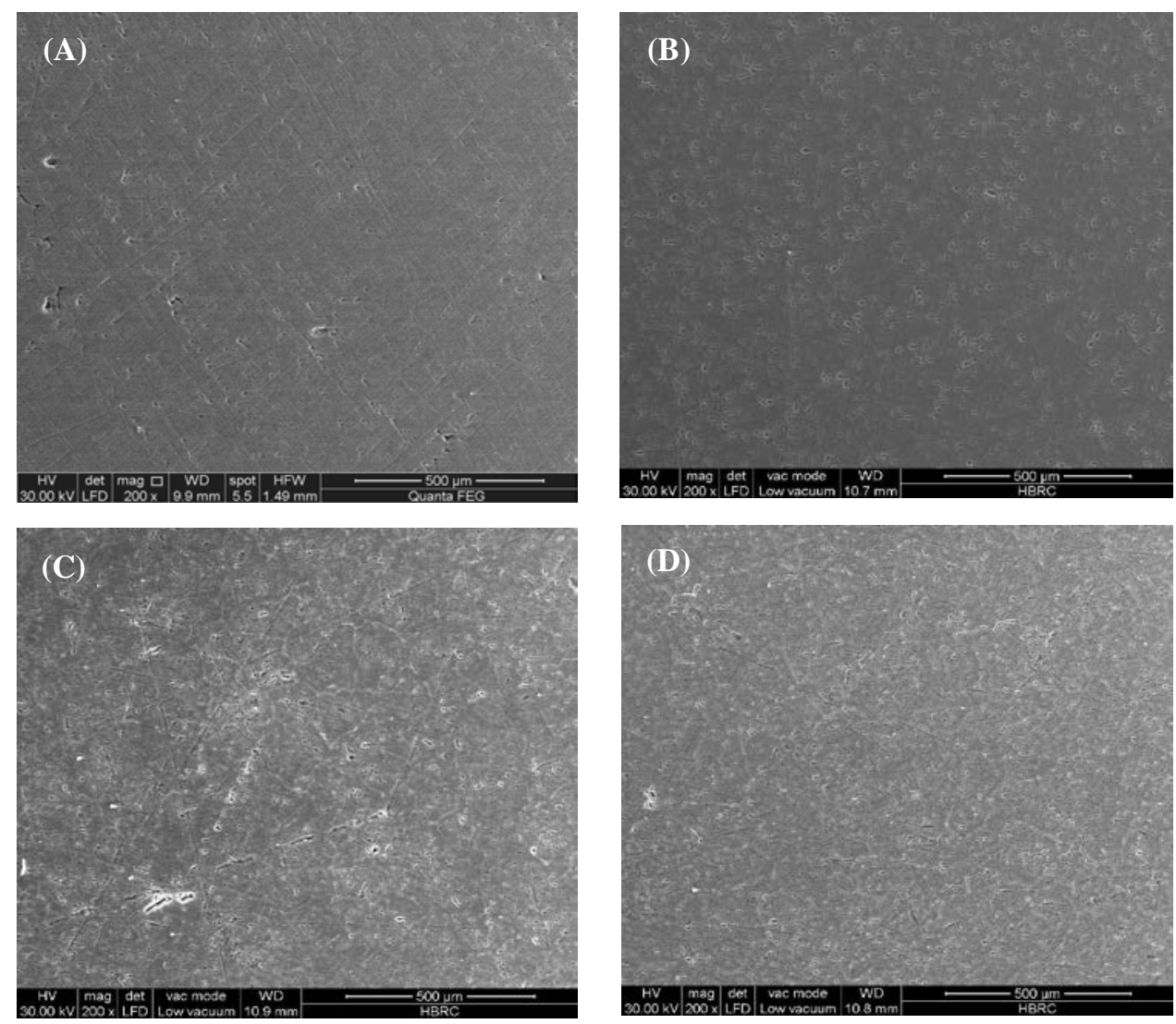

Fig.(1): SEM metallography of 6201 alloy modified with TiB and La addition in various proportions:

(a) $0.021 \mathrm{wt} \% \mathrm{TiB}_{2}$, (b) $0.0185 \mathrm{wt} \% \mathrm{La}$, (c) $0.037 \mathrm{wt} \% \mathrm{La}$,and (d) $0.0109 \mathrm{wt} \% \mathrm{La}$ respectively. 
XRD and EDS were then performed to identify the present phase and their chemical compositions are shown in Figs. (2 and 3) respectively. Fig. (2) displayed the resulting diffraction pattern recorded from the (6201alloy $+0.021 \mathrm{wt} \% \mathrm{TiB}$ ) and (6201 alloy+0.0185wt\%La). Due to the low content of Mg, Si, La and TiB in these alloys, it was not possible to observe the compositional analyzes of various phases, as reported in previous results [13]. It was found that the phase present in all diffraction patterns was Al (Reference Code: 01-072-3440). According to the results of EDS show the $\mathrm{Al}, \mathrm{Mg}$ and La elements in the (6201 alloy+0.037wt\% $\mathrm{La}$ ), while $\mathrm{Al}$ and $\mathrm{Mg}$ are observed in (6201alloy+0.021wt\%TiB) as explained before [13].

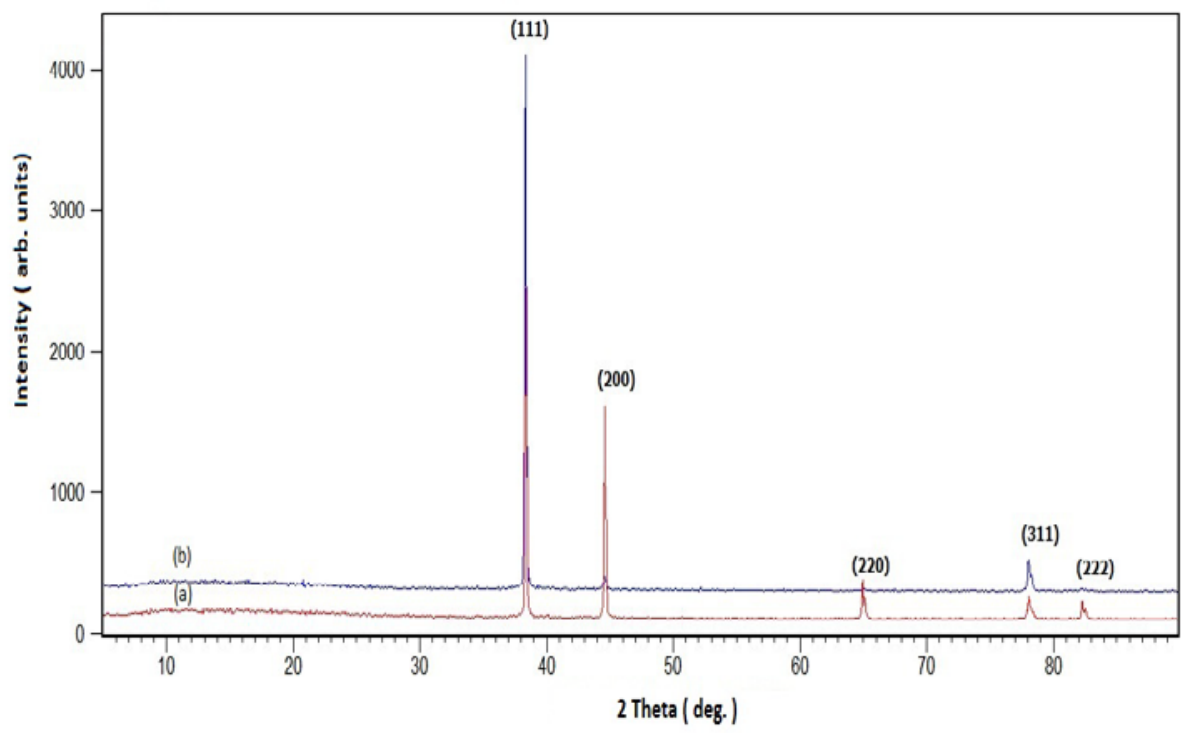

Fig. (2): X-ray diffraction patterns of (a) 6201alloy+0.021wt\%TiB and (b) 6201 alloy+0.0185wt\%La

\section{2. Analysis of Mechanical, Electrical and Ultrasonic Properties}

Experimental values of electrical resistivity, $\rho$ and Vickers microhardess, $\mathrm{Hv}$ for 6201 alloys with addition of TiB and different values of La are listed in Table (1). The results showed that the sample alloyed with addition of $0.037 \mathrm{wt} \% \mathrm{La}$ is suitable materials for using electrical conductor, because its mechanical and electrical resistivity values are approximately closed to (6201 alloy+0.021 wt\% $\mathrm{TiB}$ ) used in electrical industry. However, the formation of fine titanium boride, $\mathrm{TiB}$ compound causes decreasing harmful effect of the transition element Ti on the conductivity. On the other hand, the harmful effects of Si on the electrical conductivity of Al-Mg-Si aluminum alloys are reduced by adding $0.037 \mathrm{wt} \% \mathrm{La}$ which could form phases containing $\mathrm{La}$ and $\mathrm{Si}$ in the matrix [2,8]. This is referred to the addition of an element to another results in the formation of a compound. The effect of this compound on the electrical resistivity of a metal is far less significant than that when the alloying element is in solid solution [14]. It was also 
found that $0.0185 \mathrm{wt} \%$ amount of La addition has high strength with low conductivity. Meanwhile, 0.0109wt\% La addition has low strength with high conductivity. However, the electric alresistivity of alloys increases with the amount of elements in solid solution [2,14]. While, the strength and hardness of some metallic alloys may be enhanced by the formation of extremely small uniformly dispersed particles of a second phase within the matrix [4]. Hence, alloying elements and some available impurities in the base metal increase strength of the alloy but cause a decrease in conductivity. Therefore, the problem of high tensile with low electric conductivity is still unsolved [15]. So when selecting an aluminum alloy for using in overhead conductors, it is necessary to achieve a compromise between mechanical performance and electrical conductivity.

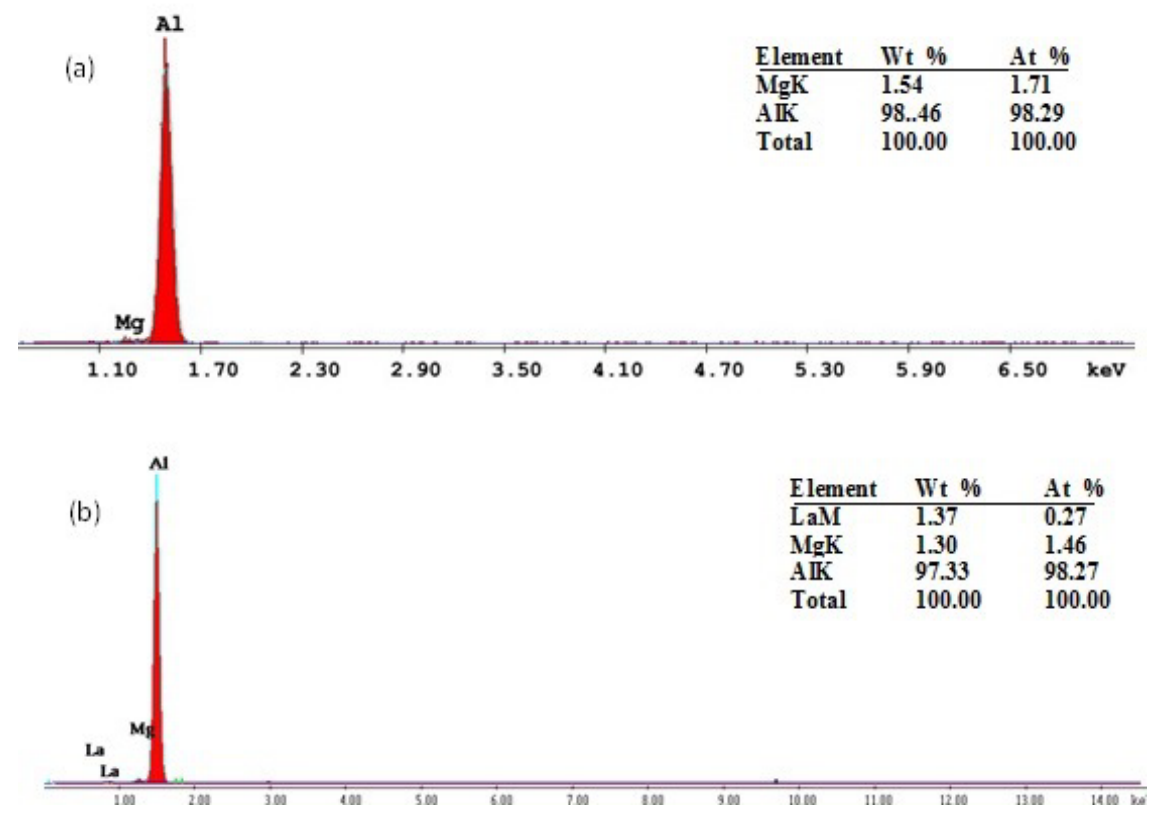

Fig. (3): EDS of 6201 alloy with (a) $0.021 w t \% \mathrm{TiB}$ and (b) $0.037 \mathrm{wt} \%$ Laudations.

Table (1): Variation of electrical resistivity ( $\rho$ ) and Vickers Microhardness (Hv) for 6201 alloys with varying contents of $\mathrm{La}$ and $\mathrm{TiB}$ respectively

\begin{tabular}{|l|l|l|}
\hline Alloy & $\rho(\Omega . \mathrm{cm})$ & $\mathrm{Hv}\left(\mathrm{N} / \mathrm{mm}^{2}\right)$ \\
\hline $0.021 \mathrm{TiB}$ & 0.141 & 57.68 \\
\hline $0.037 \mathrm{La}$ & 0.132 & 55.41 \\
\hline $0.0185 \mathrm{La}$ & 0.317 & 75.8 \\
\hline $0.0109 \mathrm{La}$ & 0.181 & 61.82 \\
\hline
\end{tabular}

Density, ultrasonic wave velocities ((longitudinal \& shear), elastic module (Young's modulus, shear modulus, longitudinal modulus, bulk modulus), Poisson's ratio and microhardness for 6201 alloys with varying contents of La and 
TiB respectively are listed in Table (2). It was found that the density of (6201 alloy+0.0185 La) was to be slightly higher than that of the (6201alloy +0.0109 La) while the density of (6201alloy+ $0.037 \mathrm{La}$ ) was the smallest density. The densities of precipitates play an important role in the ultrasonic wave, as agreement in previous results [16]. However, the density of precipitated phases is determined by the chemical composition of the alloy, so the ultrasonic wave velocities (longitudinal \& shear) values dependence on the density of precipitated phases which are contributed to form in the connectivity network structure.

Table (2): Variation of Density (D), Longitudinal $\left(V_{L}\right)$, Shear $\left(V_{s}\right)$ ultrasonic velocities, Young's modulus (E), Bulk modulus (K), Poisson's ratio $(\sigma)$ and Microhardness $(\mathrm{H})$ for 6201 alloys with varying contents of $\mathrm{La}$ and $\mathrm{TiB}$ respectively

\begin{tabular}{|l|l|l|l|l|l|l|l|}
\hline Alloy & $\mathrm{D}, \mathrm{g} / \mathrm{cm}^{3}$ & $\mathrm{~V}_{\mathrm{L}}(\mathrm{m} / \mathrm{s})$ & $\mathrm{V}_{\mathrm{S}}(\mathrm{m} / \mathrm{s})$ & $\mathrm{E}(\mathrm{Gpa})$ & $\mathrm{K}(\mathrm{Gpa})$ & $\sigma$ & $\mathrm{H}(\mathrm{Gpa})$ \\
\hline $0.021 \mathrm{TiB}$ & 2.70 & 6468 & 3237 & 75.418 & 75.234 & 0.333 & 3.152 \\
\hline $0.037 \mathrm{La}$ & 2.69 & 6074 & 3003 & 64.925 & 66.898 & 0.3382 & 2.616 \\
\hline $0.0185 \mathrm{La}$ & 2.696 & 66557 & 3243 & 75.881 & 78.107 & 0.338 & 3.060 \\
\hline $0.0109 \mathrm{La}$ & 2.695 & 6379 & 3155 & 71.79 & 73.896 & 0.3381 & 2.895 \\
\hline
\end{tabular}

\section{Conclusions}

The experimental results indicated clearly an influence of La and TiB on the microstructure, microhardness and ultrasonic parameter of 6201 alloy. However, the balance between the electrical conductivity and microhardness of the investigated 6201 alloy is influenced by both the kind and the amount of additions .It was found that 6201 alloy modifted with $0.037 \mathrm{wt} \% \mathrm{La}$ is suitable materials for using electrical conductor than the other concentrations of La. It was also found that electrical resistivity and Vickers microhardness (Hv) measured mechanically for the 6201 alloy content La had the same trend as those determined from ultrasonically.

\section{Acknowledgments}

The authors would like to express their deepest gratitude to the members and workers in the Egyptian Company of Cables and Egypt alum Compant (NagHammadt) for their facilities and valuable help, Particulary Dr. Ezzat AbdElrahman El-Katatny for his technical assistance during the preparation of the alloys. 


\section{References:}

1. P. Lamarâo,C.Santana, K. Kamizono, I. Jorge, solidified, Mater, Sci, 20, 262 (2014).

2. S. Karabay, Mater Des, 27, 821 (2006).

3. S. Karabay, Mater Des, 29, 1364 (2008).

4. G. Al-Marahleh, American journal of applied Sciences, 3(5), 1819 (2006).

5. W. Liping, G. Erjun, M. Baoxia, J. rare earth, 26, 105 (2008).

6. D. P. Mondal, N. Jha, A. Badkul, S. Das, Nonferrous Met. Soc. China, 22, 1001 (2012).

7. H. Xin, Y. Hong, J. of Wuhan University of Technology-Mater Sci Ed, 28, 202 (2013).

8. W. Yuan, Z. Liang, C. Zhang, L. Wei, Mater Des, 34, 788 (2012).

9. C. H. gür, B. Ogel, Mater Charact, 47, 227 (2001).

10. S. H. Tang, J. W. Hao, H. L. Pan, NDT\&E International, 40, 486 (2007).

11. H.Afifi,S. Marzouk, Mater Chem Phys, 80, 517 (2003).

12. F. M. Helaly, S.H. El-Sabbagh, L. Abd-El-latif, Polym Polym compos, 13(3), 1 (2005).

13. S. DelaChapelle, P. Duval, Texture Microstruct, 35(2), 55 (2002).

14. P. L. Mangonon, Ph. D., P. E., FASM, (Florida Institute Techology Melbourne) P.93 (1999).

15. T.G. Zhou, Z.Y. Jiang, J. L. Wen, H. Li, A. K. Tieu, Mater Sci, Eng, A, 485, 108 (2008).

16. C. H. Gür, I. Yildiz, J. Non destruct Eval, 27, 99 (2008). 